\section{Colorectal cancer screening in renal transplant recipients is not always cost-effective}

Regular fecal occult blood test (FOBT) screening for colorectal cancer is cost-effective in the general population, yet data are limited regarding the cost-effectiveness of FOBT screening in renal transplant recipients. To investigate this issue, Wong et al. developed a Markov model that compared annual FOBT screening with no screening in a hypothetical cohort of renal transplant recipients aged $50-70$ years.

The model simulated all the potential paths of colorectal cancer progression and incorporated probability estimates for the transitions between health states (obtained from literature searches), cancer prevalence and survival data (obtained from the Australia and New Zealand Dialysis and Transplant Registry), and data on direct health-care costs. Data that were unavailable for the renal transplant population were extrapolated from the general population. The model showed that a high level of participation in the screening program did not necessarily result in better cost-effectiveness, as the extra benefits of greater participation were canceled out by the high cost of screening a large population. Screening was slightly more beneficial for younger transplant recipients than for older recipients because younger patients are less likely to die from other competing causes. The most influential variable in the model, however, was specificity of the FOBT - a $1 \%$ change in test specificity varied the incremental cost-effectiveness ratio by 50-100\%. Overall, the model showed that annual FOBT screening is cost-effective in renal transplant recipients only under the most favorable conditions.

Original article Wong G et al. (2008) Cost-effectiveness of colorectal cancer screening in renal transplant recipients. Transplantation 85: 532-541

\section{Patients with AA nephropathy are at high risk of late-onset bladder carcinoma}

Aristolochic acid (AA) nephropathy-which is characterized by rapidly progressive kidney failure secondary to interstitial renal fibrosis - is caused by the intake of AA compounds, which are present in herbal supplements that contain Aristolochia sp. In addition to being nephrotoxic, AAs are carcinogenic, and patients with AA nephropathy are predisposed to the development of upper-tract urothelial carcinoma. In a recent paper, researchers from the Erasme Hospital, Brussels, Belgium, have reported their 15-year experience of the development of upper-tract and bladder urothelial carcinoma in a cohort of 38 women who had undergone kidney transplantations for end-stage AA nephropathy.

On ureterobinephrectomy, the researchers identified upper-tract urothelial carcinoma in 17 patients. During the 15-year follow-up period, 15 cases of bladder cancer were diagnosed, 12 of which occurred among those patients with a history of upper-tract urothelial carcinoma; the risk of developing bladder cancer was significantly greater in patients with a history of upper-tract carcinoma than in those without such a history $(P<0.01)$. Notably, patients who developed upper-tract urothelial carcinoma had ingested a significantly higher mean cumulative dose of AA than the patients who were free of cancer at the time of ureterobinephrectomy $(P<0.01)$. No significant association was found between cumulative dose of AA and development of bladder carcinoma, but this could be because of the small sample size studied.

In conclusion, the authors state that individuals exposed to AAs are at high risk of upper-tract and bladder urothelial carcinoma even 15 years after last intake. They recommend that patients with end-stage AA nephropathy should undergo bilateral ureteronephrectomy and regular cystoscopy.

Original article Lemy A et al. (2008) Late onset of bladder urothelial carcinoma after kidney transplantation for endstage aristolochic acid nephropathy: a case series with 15-year follow-up. Am J Kidney Dis 51: 471-477

\section{Nocturnal hemodialysis might improve fertility of women on renal replacement therapy}

In comparison with women in the general population, women on conventional hemodialysis have decreased fertility and a higher incidence of fetal mortality. Barua et al. have suggested that uremia-related changes might partially explain these poor outcomes. They 